\title{
BOTHRIURUS PICHICUY, NUEVO ESCORPIÓN CHILENO DEL GRUPO VITTATUS (SCORPIONES, BOTHRIURIDAE)
}

Camilo I. Mattoni ${ }^{1,2}$

\begin{abstract}
BOTHRIURUS PICHICUY, NEW CHILEAN SCORPION FROM THE VITTATUS GROUP (SCORPIONES, BOTHRIURIDAE). Bothriurus pichicuy, a new scorpion species from the center coast of Chile, is described. It belongs to the vittatus species-group, and differs from the others species in the group by the pigmentation patterns of the prosoma, mesosoma and venter of metasoma, by the development and arrangement of the ventral keels of caudal segment $\mathrm{V}$, and for its small size. The species is the only representative of the vittatus species-group that lives in sympatry with another Bothriurus species: B. coriaceus Pocock, 1893, from the coastal desert of Chile. Records of B. pichicuy came from the provinces of Petorca and Choapa.
\end{abstract}

KEYWORDS. Bothriurus, Scorpiones, Bothriuridae, Chile, Neotropical.

\section{INTRODUCCIÓN}

La escorpiofauna de Chile ha recibido poca atención en los últimos tiempos, luego de varios trabajos de las décadas del 1960, 1970 y principios de los 1980 (CEKALOVIC, 1966; 1974; 1983; San Martín \& Cekalovic, 1968), se han realizado pocas contribuciones recientemente (AcostA, 1998; MATtONI, 2002). Las investigaciones sobre el género Bothriurus Peters, 1861 son escasas, y muchas de las especies citadas para Chile son de identidad dudosa. A esto contribuyó, probablemente, el desconocimiento de los materiales típicos de las especies por parte de los investigadores, incluso especies muy antiguas son poco conocidas (por ejemplo, B. coriaceus Pocock, 1893) y necesitan de una redescripción detallada y una actualización de sus áreas de distribución. Por éste motivo, y además para una revisión del género Bothriurus y estudio de la relaciones filogenéticas entre las especies, emprendimos un detallado estudio de las especies de Chile. Entre la gran cantidad de ejemplares estudiados, observamos un grupo de especies muy relacionadas: el grupo vittatus (MATTONI, 2002); en el que las especies muestran una morfología del hemiespermatóforo idéntica, con dos apófisis conspicuas en la cara externa del lóbulo interno, y una disposición de las carenas ventrales del segmento caudal I similar. El grupo comprende cuatro especies: Bothriurus vittatus (Guérin Meneville, 1838),

1. Cátedra de Diversidad Animal I, Facultad de Ciencias Exactas, Físicas y Naturales, Universidad Nacional de Córdoba, Av. Vélez Sarsfield 299 , 5000 Córdoba, Argentina. (cmattoni@com.uncor.edu)

2. Becario de CONICET.

Iheringia, Sér. Zool., Porto Alegre, 92(4):81-87, 30 de dezembro 2002 
B. picunche Mattoni, 2002 y dos formas innominadas (MATTONI, 2002). Las especies presentan una distribución alopátrica, desde el sur de la Región de Coquimbo hasta la Región de La Araucanía; se describe una especie nueva que representa hasta el momento el límite norte del grupo.

\title{
MATERIAL Y MÉTODOS
}

La terminología empleada sigue a la aplicada por STAHNKE (1970), excepto por los términos referidos a las carenas del metasoma y los pedipalpos (FRANCKE, 1977) y la nomenclatura de las tricobotrias (VACHON, 1974). Para las estructuras del hemiespermatóforo se utilizan las propuestas por SAN MARTíN (1963). Las medidas están expresadas en milímetros (L, largo; A, ancho; H, alto). Se utilizan las siguientes abreviaturas de las carenas del metasoma: Dl, dorsales laterales; Lsm, laterales supramedianas; Lm, laterales medianas; Lim, laterales inframedianas; Vl, ventrales laterales; Vsm, ventrales submedianas; Vm, ventral mediana; y del hemiespermatóforo: L, lámina; cd, cresta distal de la lámina; rdp, repliegue distal posterior; li, lóbulo interno; lb, lóbulo basal; le, lóbulo externo.

Los especímenes utilizados provienen de las siguientes colecciones (curadores entre paréntesis): MACN, Museo Argentino de Ciencias Naturales "Bernardino Rivadavia", Buenos Aires (C. Scioscia); CDA, Cátedra de Diversidad Animal I, Facultad de Ciencias Exactas, Físicas y Naturales, Universidad Nacional de Córdoba, Argentina (L. Acosta); UCCC, Universidad de Concepción Colecciones Científicas, Chile (J. Artigas).

\section{Bothriurus pichicuy sp. nov.}

\author{
(Figs. 1-12)
}

Holotipo $\sigma^{-2}$, de CHILE, Región de Coquimbo (V): Provincia Petorca, Pichicuy, Quebrada

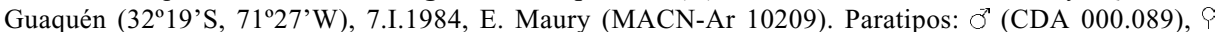
(MACN-Ar 10210), 2 juveniles (CDA 000.090, MACN-Ar 10211), mismos datos del holotipo; Región

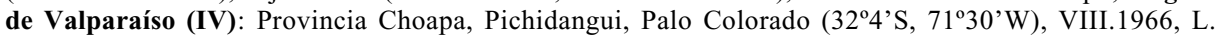
Peña, 9 (UCCC 4274). típica.

Etimología. El nombre específico es un sustantivo en aposición y refiere a la localidad

Distribución. Conocida sólo del extremo costero sur de la Provincia de Choapa y de la costa norte de la Provincia de Petorca, en Chile.

Diagnosis. Se distingue de las otras especies del grupo vittatus por su tamaño (machos: 23,01-28,23 mm; hembras: 21,62-30 $\mathrm{mm}$ ) y por sus patrones de pigmentación, al ser la única especie que presenta las tres bandas ventrales de pigmento negro del metasoma no confluentes en ningún segmento (fig. 12) (siempre confluyen en algún segmento en las otras especies), así como las bandas ventrales laterales discontinuas en todos los segmentos del metasoma (fig. 12) (contínuas en las otras especies). La abundante granulación ventral del segmento caudal V (fig. 2) (casi uniforme en todo el segmento, más destacada en los machos) también la distingue de las restantes especies, donde la granulación es menos abundante y jamás alcanza tan marcadamente la porción anterior del segmento.

Descripción. Coloración (en alcohol) general amarillo rojizo, con manchas de pigmento marrón obscuro y negro. Patas amarillentas con manchas difusas, más notables en prolateral. Coxas y pectenes amarillos, sin manchas. Pedipalpos amarillo rojizo, con manchas difusas, más marcadas en dorsal y retrolateral. Prosoma (fig. 10) con cúpula ocular casi negra, área central con mancha trapezoidal conectada con manchas irregulares laterales y con sector de ojos laterales, área central anterior con mancha transversal, 
región posterior con un par de manchas irregulares en los ángulos que llegan casi al borde posterior del prosoma. Tergitos (fig. 11) amarillo rojizo, pretergito casi totalmente manchado, área central sin pigmento, formando ancha banda clara, áreas laterales muy manchadas, área paramediana posterior con pigmento más difuso. Esternitos: amarillentos, I-IV sin manchas, $\mathrm{V}$ con dos manchas laterales y una banda central angosta. Metasoma amarillento rojizo, por ventral (fig. 12) tres bandas de pigmento no confluentes, la central delgada y contínua en cada segmento, las laterales delgadas en proximal, anchas en distal, discontínuas; en segmentos I-IV por dorsal par de pequeñas manchas triangulares centrales y manchas irregulares en sectores lateroposteriores de cada segmento caudal, por dorsal del segmento $\mathrm{V}$ par de manchas laterales longitudinales. Telson rojo amarillento, manchado con pigmento tenue, por ventral, par de estrechas líneas longitudinales paramedianas claras, por lateral una línea clara, por dorsal amarillento (sin manchas en machos, hembras con débil pigmentación). Morfología: carenas de mayor desarrollo en los machos, excepto por las Vsm y Vl del segmento caudal I y esternito V, ausentes en los machos y desarrolladas en las hembras. Prosoma y tergitos de tegumento finamente granuloso en machos, liso en hembras. Prosoma: margen anterior levemente escotado (casi recto), surco mediano anterior muy poco marcado, cúpula ocular prominente y sin surco, surco mediano central bien marcado y foseta profunda, surcos transversales centrales y posteriores laterales poco marcados, surcos transversales posteriores marcados, ojos medios separados un diámetro. Tergitos: I-VI sin carenas, VII con cuatro carenas cortas en el tercio posterior, dos paramedianas y dos laterales, con gránulos dispersos entre ellas (sin granos entre paramedianas). Esternitos: tegumento con granulación muy fina, en aumento desde el I al V (más notable en machos), esternito $\mathrm{V}$ con cuatro carenas vestigiales (dos paramedianas, dos laterales) en el cuarto posterior (ausentes en machos). Metasoma: carenas de segmentos I-IV, Dl completas, débiles (especialmente en segmento I), de granos bajos y redondeados, los granos posteriores un poco más desarrollados; Lsm presentes sólo en el tercio posterior de cada segmento; área entre carenas Dl y Lsm con algunos gránulos pequeños dispersos; Lim sólo presentes en el tercio distal de segmentos I-III (muy débiles en segmentos II y III), ausentes en segmento IV; Vl y Vsm presentes sólo en segmento I de las hembras, irregulares, vestigiales (sin granos), Vsm bifurcada anteriormente, área entre Vsm con granos chatos medianos, en machos superficie ventral de segmento caudal I con granos pequeños; carenas de segmento caudal V (figs. 1, 2, 5): Dl ausente, Lm ausente, área entre Dl y Vl con granulación irregular (granos grandes y bajos); Vl, Vsm y Vm desarrolladas en la mitad distal del segmento, Vsm ligeramente oblicuas, Vm bifurcada hacia distal; toda el área ventral con abundantes granos romos y grandes; par anterior de macrosetas ventromediales presente. Telson (figs. 1, 5) vesícula alargada (un poco menos en las hembras), superficie ventral con gránulos romos, dorsal lisa (con leve depresión glandular en machos). Quelíceros con dos dientes subdistales en el dedo móvil. Pedipalpos: fémur con carenas dorsoexterna, dorsointerna y ventrointerna desarrolladas en la mitad proximal, con gránulos redondeados, cara interna con gránulos dispersos; patela con dos carenas, dorsointerna y ventrointerna, muy débiles (vestigiales); pinzas sin carenas, sólo se observan vestigios de carenas en los dedos; pinza del macho (figs. 3, 4) robusta, de dedos cortos, con fuerte apófisis cónica en faz interna, próxima a la base del dedo móvil, y con depresión suave sobre la apófisis, tegumento con granulación muy fina (granos más notables en la depresión interna); pinza de la hembra (figs. 6, 7) menos robusta, de dedos proporcionalmente un poco más largos, 
con un gránulo romo en la posición de la apófisis del macho, sin depresión, tegumento liso, con algunos granos pequeños en la cara interna de la base del dedo fijo. Tricobotriotaxia: tipo $\mathrm{C}$, neobotriotaxia aumentadora, con los siguientes totales por segmento: fémur $3(1 d ; 1 i ; 1 e)$, patela $19(2 d ; 1 i ; 13 e ; 3 v)$ y pinza $27(21$ en mano $-5 V$; 6 en dedo fijo). Número de dientes pectíneos: holotipo macho 16-16 (ver variabilidad debajo). Hemiespermatóforo (figs. 8, 9): L angosta, suavemente curvada en S; cd paralela al borde de la lámina, dividida en dos por un tabique transversal, porción distal levemente curva, porción proximal recta; lb laminar, bífido en su extremo; li con dos apófisis en la faz externa; le con un tabique semicircular; rdp bien desarrollado.

Medidas del macho holotipo: L total 23,01. Prosoma: L/A anterior 2,93/1,9. L mesosoma 6,1. Metasoma: L total 13,98; L/A segmento I 1,23/2,1; L/A II 1,6/2; L/A III 1,93/ 1,96; L/A IV 2,23/1,93; L/A/H V 2,96/1,7/1,43; L/A/H telson 4,03/1,5/1,07, L aguijón 1,33, L vesícula 2,7. Pedipalpo derecho: $\mathrm{L}$ total 10,14; $\mathrm{L}$ trocánter 0,93; L/A fémur 2,23/0,87; L/A patela 2,36/0,93; L/A/H pinza 4,62/1,73/2,03; L dedo móvil 2,38. Hembra paratipo (MACNAr 10210): $\mathrm{L}$ total 21,62. Prosoma: $\mathrm{L} / \mathrm{A}$ anterior 2,80/2. L mesosoma 6,38. Metasoma: $\mathrm{L}$ total 12,44; L/A segmento I 1,2/2,11; L/A II 1,49/1,98; L/A III 1,68/1,91; L/A IV 1,94/1,88; L/A/H V 2,59/1,81/1,46; L/A/H telson 3,54/1,3/1,04, L aguijón 1,23, L vesícula 2,31. Pedipalpo derecho: $\mathrm{L}$ total 9,3; $\mathrm{L}$ trocánter 0,$78 ; \mathrm{L} / \mathrm{A}$ fémur 2,01/0,84; L/A patela 2,17/0,94; L/A/H pinza 4,34/1,3/1,59; L dedo móvil 2,27.

Variabilidad. Largo total, machos: 23,01-28,23 (n=2); hembras: 21,62-30 (n=2). Número de dientes pectíneos: machos 15 (2 pectenes), 16 (2); hembras 14 (4). La pigmentación varía ligeramente entre los ejemplares pero siempre manteniendo el patrón descripto, excepto en una hembra (Palo Colorado) en la que las tres franjas ventrales del segmento caudal V muestran un esbozo de confluencia entre ellas hacia distal del segmento. El macho paratipo (CDA) presenta el aguijón del telson casi recto, alineado con la superficie dorsal del telson, al parecer una malformación.

Discusión. El número de dientes pectíneos, de uso frecuente para distinguir especies de escorpiones, no presenta por ahora utilidad en la sistemática de las especies del grupo vittatus. Inversamente, los patrones de pigmentación son extremadamente útiles (además de algunos caracteres morfológicos) para la distinción de las especies dentro del grupo, ya que muestran patrones diferentes. Se observa entre las especies un decrecimiento en la cantidad de pigmento en el metasoma de sur a norte de Chile: $B$. vittatus (Regiones de La Araucanía y Bío Bío) presenta las tres bandas de pigmento ventrales del metasoma contínuas, gruesas y confluentes hacia distal de cada segmento; B. picunche (sectores próximos a la costa de Regiones de Maule y O'Higgins, MATTONI, 2002) tiene bandas de pigmento contínuas, más delgadas y confluentes sólo en el segmento V; mientrás que $B$. pichicuy muestra las bandas más reducidas: discontínuas (las laterales), delgadas y nunca confluentes. La vegetación en Chile presenta un marcado gradualismo latitudinal: desde bosques húmedos (sur) hasta formaciones desérticas (norte) (GAJARDO, 1995), y el patrón de disminución de pigmento en el grupo vittatus de sur a norte estaría en concordancia con lo observado por LouREnço \& CloudsLey-Thompson (1996), que mencionan que especies de zonas desérticas o más áridas presentan una menor cantidad de pigmento que las provenientes de regiones más humedas y forestadas.

Por el momento, éste es el único Bothriurus de Chile que vive en simpatría con otra especie del género, Bothriurus coriaceus, que ha sido registrada también de Quebrada Guaquén (C. Mattoni y L. Acosta, datos inéditos). Bothriurus coriaceus ocurre en las 


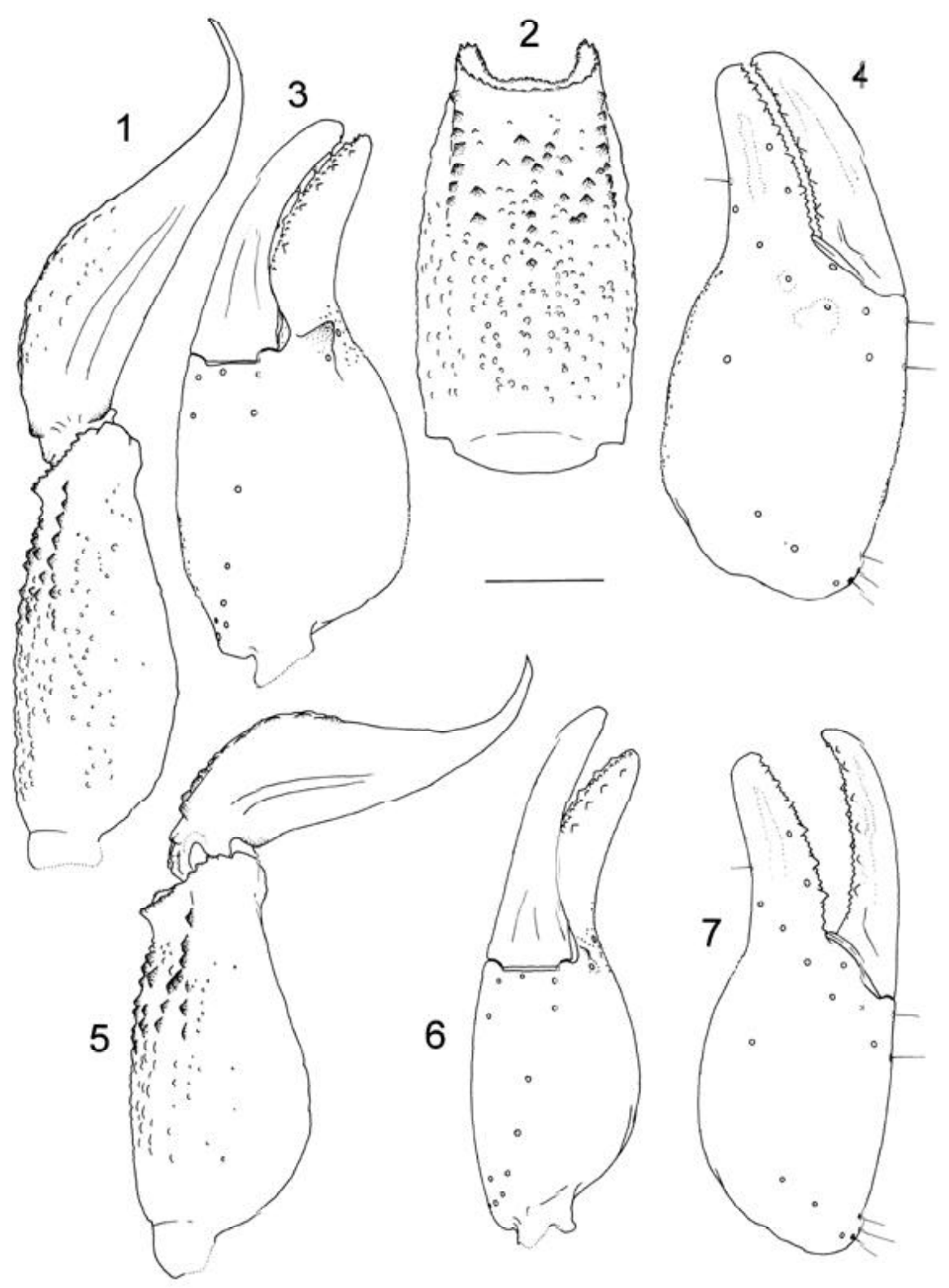

Figs. 1-7. Bothriurus pichicuy sp. nov. 1-4, holotipo $\sigma^{-1}$ (MACN): 1, segmento caudal $\mathrm{V}$ y telson, vista lateral; 2, segmento caudal V, vista ventral; 3, pinza derecha, vista ventromedial; 4, vista externa; 5-7, paratipo 9 (MACN-Ar 10210): 5, segmento caudal V y telson, vista lateral; 6 , pinza derecha, vista ventromedial; 7, vista externa. Escala: $1 \mathrm{~mm}$. 


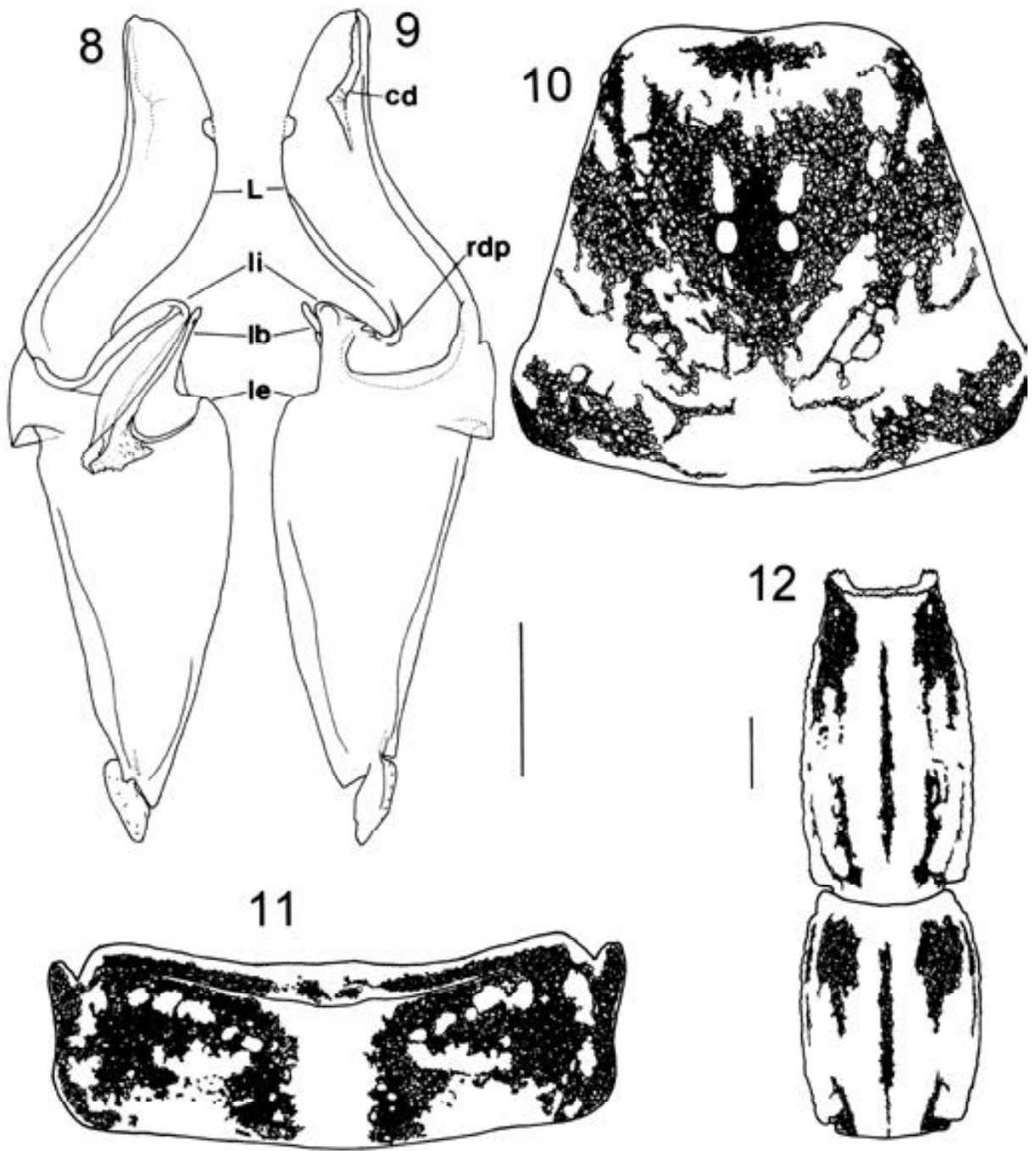

Figs. 8-12. Bothriurus pichicuy sp. nov. 8-10, holotipo (MACN): 8, hemiespermatóforo izquierdo, vista interna; 9, vista externa; 10, prosoma; paratipo (MACN-Ar 10210): 11, tergito IV; paratipo 0 (CDA 000.089): 12, segmentos caudales IV y V, vista ventral. (Abreviaturas: L, lámina; cd, cresta distal de la lámina; rdp, repliegue distal posterior; li, lóbulo interno; lb, lóbulo basal; le, lóbulo externo). Escalas: $1 \mathrm{~mm}$; $8-11$ a la misma escala.

Iheringia, Sér. Zool., Porto Alegre, 92 (4):81-87, 30 de dezembro 2002 
subregiones del Desierto Costero y del Matorral Estepario (GAJARDO, 1995). Es en ésta última zona donde $B$. pichicuy y $B$. coriaceus conviven. Ésta región tiene una precipitación muy baja e irregular, y el predominio en toda el área de $B$. coriaceus (registrado en muchas localidades, C. Mattoni y L. Acosta, datos inéditos) reafirma su carácter de especie adaptada a zonas muy secas. Mientrás que las especies conocidas del grupo vittatus habitan todas en sectores más húmedos del centro y sur de Chile (MATTONI, 2002), y la presencia de una especie perteneciente a éste grupo en regiones más áridas llama la atención. Quizás B. pichicuy demuestre una suerte de "relicto" de una antigua extensión mucho mayor del grupo, limitada ahora por condiciones ecológicas. A favor de ésta hipótesis estaría el hecho de que uno de los lugares de captura (Quebrada Guaquén) es un sector un poco más húmedo que sus alrededores (P. Goloboff, comunic. personal), explicando de cierto modo la distribución tan restricta de la especie.

Agradecimientos. A los curadores de las diversas instituciones por el préstamo del material examinado, y a L. E. Acosta por su constante ayuda. Este estudio fue parcialmente financiado con fondos otorgados a L. E. Acosta por la Secretaría de Ciencia y Técnica de la Universidad Nacional de Córdoba (SECYT, UNC), y forma parte de mi Tesis Doctoral en la Universidad de Córdoba bajo la supervisión de L. E. Acosta.

\section{REFERENCIAS BIBLIOGRÁFICAS}

Acosta, L. E. 1998. Urophonius transandinus sp. nov. (Bothriuridae), a scorpion from central Chile. Stud. Neotrop. Fauna Environm., Lisse, 33:157-164.

Cekalovic, T. K. 1966. Contribución al conocimiento de los escorpiones chilenos. Not. Mens. Mus. Nac. Hist. Nat. Santiago, Santiago, 10(118):1-8.

1974. Bothriurus dumayi n. sp. de escorpión chileno (Scorpiones, Bothriuridae). Boln Soc. Biol. Concepción, Concepción, 48:209-216.

1983. Catálogo de los escorpiones de Chile (Chelicerata, Scorpiones). Boln Soc. Biol. Concepción, Concepción, 54:43-70.

Francke, O. F. 1977. Two enmendations to Stahnke's (1974) Vaejovidae revision (Scorpionida, Vaejovidae). J. Arachnol., Texas, 4:125-135.

Gajardo, R. 1995. La vegetación natural de Chile. Clasificación y distribución geográfica. Santiago, Universitaria. $165 \mathrm{p}$.

Lourenço, W. R. \& Cloudsley-Thompson, J. L. 1996. The evolutionary significance of colour, colour patterns and fluorescence in scorpions. Revue suisse Zool., Genève, v. hors série(2):449-458.

Mattoni, C. I. 2002. Bothriurus picunche sp. nov., a new scorpion from Chile (Bothriuridae). Stud. Neotrop. Fauna Environm., Lisse, 37(2):169-174.

San Martín, P. R. 1963. Una nueva especie de Bothriurus (Scorpiones, Bothriuridae) del Uruguay. Bull. Mus. natn. Hist. nat., Paris, $2^{\mathrm{e}}$ série, 35(4):400-418.

San Martin, P. R. \& Cekalovic, T. K. 1968. Escorpiofauna Chilena I. Bothriuridae. Una nueva especie de Urophonius para Chile. Inv. Zool. Chil., Santiago, 13:81-99.

StahnKe, H. L. 1970. Scorpion nomenclature and mensuration. Ent. News, Washington, 81:297-316.

VACHON, M. 1974. Etude des caractères utilisés pour classer les familles et les genres de scorpions (Arachnides). 1. La tricobothriotaxie en Arachnologie. Sigles trichobothriaux et types de tricobothriotaxie chez les Scorpions. Bull. Mus. natn. Hist. nat., Paris, $3^{\text {e }}$ série, 140(104):857958 .

Recebido em 07.05.2002; aceito em 30.09.2002. 